\title{
Thin Film Photoemission Experiments
}

\author{
R. Brogle, P. Muggli, and C. Joshi \\ Electrical Engineering Department \\ University of California Los Angeles, CA 90024
}

\begin{abstract}
The "promptness" of the photoemission process is an important issue in the production of ultra-short electron bunches from photoinjector guns. One would ideally hope to obtain electron emission whose duration faithfully mimics the photon pulse for photon pulses on the order $100 \mathrm{fs}$ for many advanced accelerator applications. If all the photoelectrons were emitted at the surface, the electron current would indeed mimic the photon pulse. However, it is important to experimentally determine the relative contributions of the surface versus the bulk of the material to the net photoelectron current. It is the purpose of these experiments to address this issue.
\end{abstract}

\section{PHOTOELECTRON DYNAMICS}

The standard model of volume photoemission consists of a three step process: (1) the electron absorbs a photon of energy $h \nu$ which elevates it to an excited energy state, (2) the excited electron travels to the surface, and (3) the electron crosses the surface barrier to escape the material. The height of the surface barrier above the Fermi level is $e \Phi$, where $e$ is the magnitude of the electron charge and $\Phi$ is the work function of the material. The total emitted charge will depend on the depth into which the incident light can penetrate and the depth from which a photoexcited electron can reach the surface before losing its escape energy.

For optically excited electrons in metals, the primary mechanism for energy loss will be inelastic collisions with the conduction band electrons [1]. A single such collision will on average result in a significant loss of energy for the excited electron because the conduction electrons with which it collides have much lower energies. After the collision the electron most likely will no longer have sufficient energy to overcome the surface barrier and will not be emitted ( $h \nu-$ $e \Phi<e \Phi$ for the wavelengths in this experiment). Thus the maximum electron escape depth is dependent on the range $l$ that the photoexcited electrons can travel before suffering a collision.

In general, electrons may also suffer elastic collisions with lattice phonons and may lose energy through plasmon excitation. However, for visible photon excitation energies the electron-phonon mean free path is much larger than the electron-electron mean free path. Therefore, electron-phonon collisions can be 


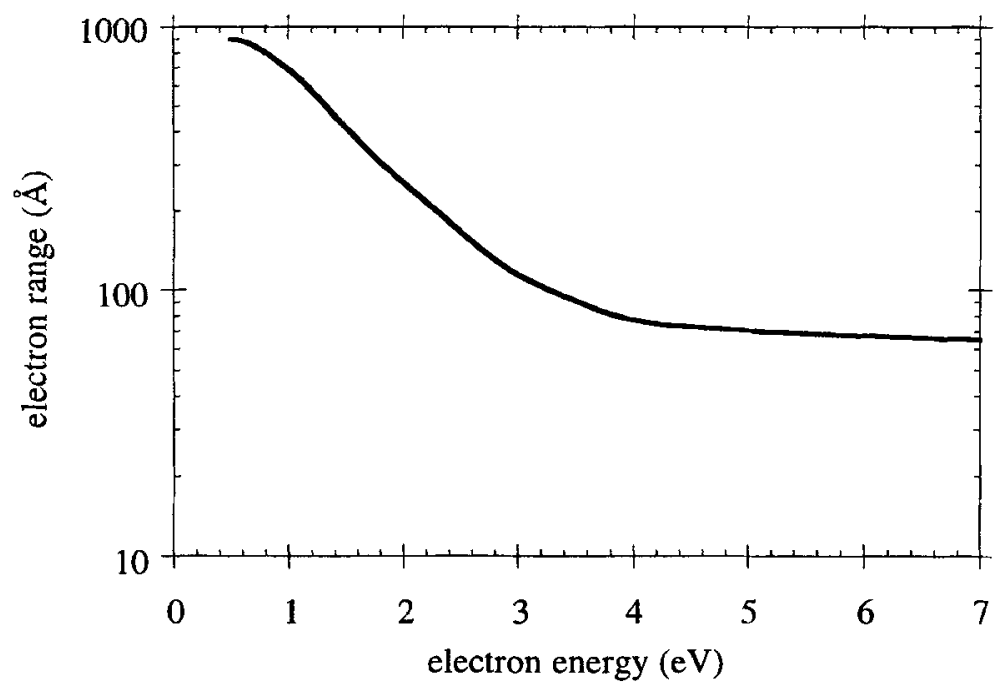

Figure 1: Empirical curve for electron range vs. excitation energy (with respect to the Fermi level) as measured in gold films (from $S z e$ et al.)

ignored. Also, the photon energies are too small to create photoelectrons capable of exciting plasma waves ( $\hbar \omega_{p} \approx 11 \mathrm{eV}$ for copper) - this effect can be ignored as well. The electron range $l$ will then be equal to the electronelectron mean free path, and any electrons which are emitted will have traveled ballistically to the surface.

At visible wavelengths, $l$ is a decreasing function of the excitation energy [2]. This can be explained in terms of the Pauli exclusion principle for the conduction electrons. A photoelectron that has been excited to an energy $\varepsilon$ (measured with respect to the the Fermi level $\epsilon_{F}$ ) can interact only with those conduction electrons having energy between $\epsilon_{F}$ and $\epsilon_{F}-\varepsilon$. Conduction electrons below this energy cannot be excited above the Fermi level because the photoelectron energy is less than this energy difference, and there are no unoccupied energy states below the Fermi level. Thus no energy exchange between the conduction electrons below $\epsilon_{F}-\varepsilon$ and the excited electron can take place. The greater the excitation energy $\varepsilon$, the larger the number of conduction electrons that the photoelectron can interact with, and therefore the shorter the electron range. Fig. 1 shows an empirical curve taken from [1] showing this behavior of the electron range $l$ in gold films.

Linear photoemission from thin metal films can be investigated using a simple one-dimensional analysis [3]. This approach is sufficient because the 


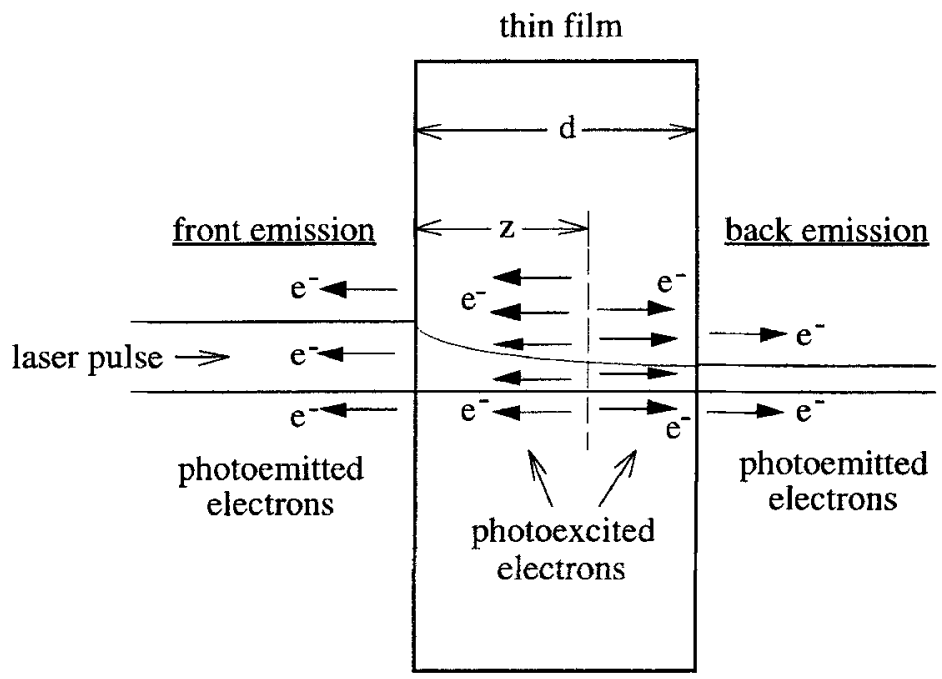

Figure 2: Front and back side photoemission from a film of thickness $d$.

isotropy of the electron velocities makes it possible to study motion in the $\hat{z}$ direction (the direction normal to the surface) independently from the electron motion in the $\hat{x}$ or $\hat{y}$ directions. In addition, for optically excited electrons most of the kinetic energy must be in the $\hat{z}$ direction in order to overcome the metal surface barrier, thus the emitted electrons will have had primarily $z$ motion in the metal.

Fig. 2 depicts a laser pulse incident on a thin metal film of thickness $d$. Let us look at electrons at a depth $z$ from the front surface (the surface onto which the laser pulse is incident). As the pulse propagates through the metal, its intensity decreases exponentially:

$$
I(z)=I_{0} e^{-z / \delta}
$$

where $\delta$ is the optical absorption depth at the laser wavelength. The probability of an electron at depth $z$ absorbing one photon is proportional to this intensity:

$$
P_{\gamma}(z) \propto I_{0} e^{-z / \delta}
$$

An electron which absorbs a photon must then travel to the front surface without suffering a collision in order to have a chance to escape. The probability $P_{n c}$ of no collision occuring over this distance $z$ is

$$
P_{n c}(z) \propto e^{-z / l}
$$

Thus the total probability for the front surface emission of an electron from a depth $z$ is

$$
P_{\text {front }}(z)=P_{\gamma}(z) \cdot P_{n c}(z) \propto I_{0} e^{-z / \delta} e^{-z / l}
$$


For a film of thickness $d$, the electrons which lie a distance $z$ from the front surface will be at a distance $d-z$ from the back surface. The probability for emission from the back surface is then

$$
P_{b a c k}(z)=P_{\gamma}(z) \cdot P_{n c}(d-z) \propto I_{0} e^{-z / \delta} e^{-(d-z) / l}
$$

To find the total electron yield from each surface, we must integrate the emission probabilities over the thickness of the film. For a given incident light intensity the emitted charge from the front is

$$
Q_{\text {front }} \propto \int_{0}^{d} I_{0} e^{-z / \delta} e^{-z / l} d z=\frac{I_{0}}{\frac{1}{\delta}+\frac{1}{l}}\left[1-e^{-\left(\frac{1}{\delta}+\frac{1}{l}\right) d}\right]
$$

and the charge emitted from the back is

$$
Q_{b a c k} \propto \int_{0}^{d} I_{0} e^{-z / \delta} e^{-(d-z) / l} d z=\frac{I_{0}}{\frac{1}{\delta}-\frac{1}{l}}\left[e^{-d / l}-e^{-d / \delta}\right]
$$

For a laser pulse with a gaussian spatial and temporal profiles the total energy $E$ in the pulse is related to the peak intensity $I_{0}$ by

$$
E=\sqrt{\pi} \tau r_{0}^{2} I_{0}
$$

where $\tau$ and $r_{0}$ are 1/e "widths" of the temporal and spatial profiles. Therefore we can express the total integrated charge from a one-photon emission process in terms of the total integrated energy of the gaussian laser pulse:

$$
Q=b E
$$

where the constant $b$ is the electron yield in units of $\mathrm{pC} / \mu \mathrm{J}$. For the front and the back side emission from a thin film the electron yields will be

$$
\begin{aligned}
& b_{\text {front }}=\frac{K}{\frac{1}{\delta}+\frac{1}{l}}\left[1-e^{-\left(\frac{1}{\delta}+\frac{1}{l}\right) d}\right] \\
& b_{b a c k}=\frac{K}{\frac{1}{\delta}-\frac{1}{l}}\left[e^{-d / l}-e^{-d / \delta}\right]
\end{aligned}
$$

where $K$ is the constant of proportionality having units of $\mathrm{pC} /(\mu \mathrm{J} \cdot \AA)$. For large thicknesses $b_{\text {front }}$

$$
\lim _{d \rightarrow \infty} b_{\text {front }}=b_{\text {bulk }}
$$

and therefore we can determine the value of $K$ from the front emission properties:

$$
K=\left(\frac{1}{\delta}+\frac{1}{l}\right) b_{b u l k}
$$




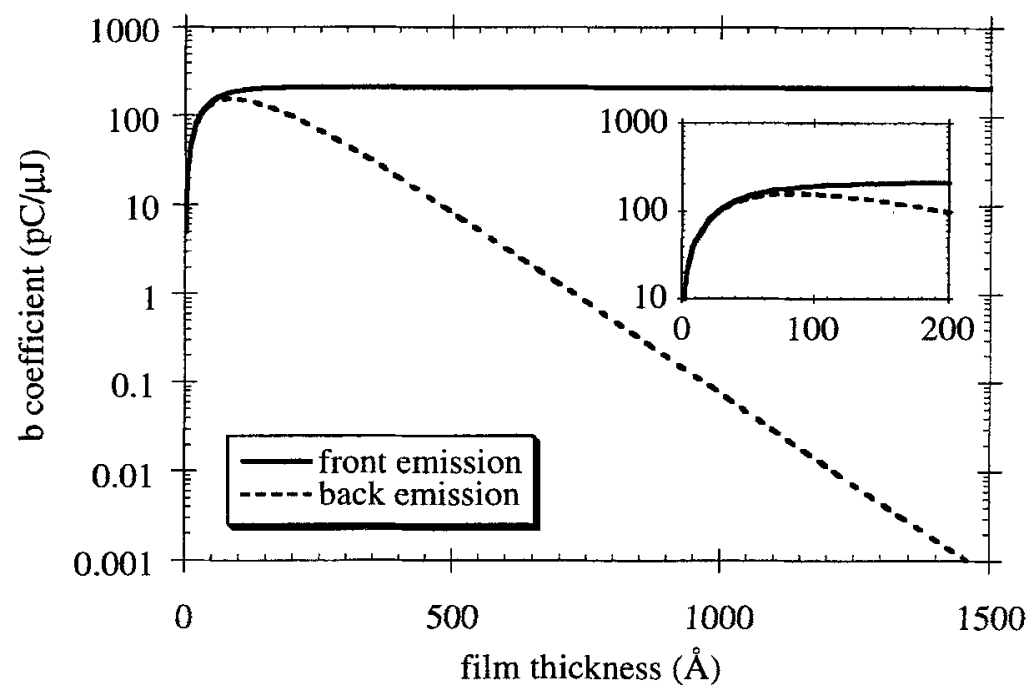

Figure 3: The theoretical behavior of the electron yield (as measured by the $b$ coefficient in Eqs. 9 and 10) vs. film thickness $d$ for front and back illumination of copper by $217 \mathrm{~nm}$ light. The optical absorption depth is taken to be $\delta_{217}=$ $105 \AA$ and the electron range is $l=70 \AA$. 
Fig. 3 shows the behavior of Eqs. 9 and 10 as a function of film thickness $d$ for $217 \mathrm{~nm}$ incident light on copper. We used the published optical absorption depth value for copper of $\delta_{217}=105 \AA[4]$ and the electron range value suggested by Fig. 1 of $l=70 \AA$ for a $5.7 \mathrm{eV}$ excitation energy. For small film thicknesses $(d<l)$ the front and back electron yields are equal and increase as the thickness becomes larger. For greater thicknesses $(d>l)$ the front yield reaches the bulk $b$ value after about $200 \AA$ and remains constant, while the back yield turns around and decays exponentially, dropping by 5 orders of magnitude over $1500 \AA$.

\section{FRONT AND BACK ILLUMINATION EXPERIMENT}

The setup for the thin film illumination experiment is shown in Fig. 4. The samples were $1^{\prime \prime} \times 2^{\prime \prime}$ fused silica slides with steps of various thicknesses of copper film evaporated onto one side. Two samples were used, one with film thicknesses ranging from $50-500 \AA$ and the other with thicknesses $500-1400 \AA$. The hollow anode was mounted on a rotation stage with an external control so that it could be positioned under vacuum on either side of the sample for the front illumination (Fig. 4a) and back illumination (Fig. 4b) configurations. A mechanical feedthrough was used to move the sample to illuminate each thickness and also to flip the slide so that the copper side would face the anode for each configuration. The transmission of the laser pulse through each thickness of copper film was measured using a photodiode placed behind the sample outside of the vacuum chamber.

The electron yield $b$ was determined for each film thickness by measuring the emitted charge $Q$ as a function of incident laser pulse energy $E$. Both the front and back side emission demonstrated the expected proportionality given in Eq. 8. Each $217 \mathrm{~nm}$ laser pulse had a duration of $400 \mathrm{fs}$, and the pulses were varied in energy from 0.001 to $1 \mu \mathrm{J}$. A plot of the front and back electron yield as a function of film thickness is shown in Fig. 5.

From the plot, we see that the front $b$ coefficient increases as the films become thicker until it reaches a bulk value of $b_{b u l k}=190 \mathrm{pC} / \mu \mathrm{J}$ at $250 \AA$. This increase is more gradual than predicted by the theory. As the films become thicker than this value we observe no additional emitted charge, thus we take $250 \AA$ to be the maximum depth $\left(d_{\max }\right)$ from which electrons can escape the metal for this incident wavelength. Note that the front emission from thicknesses $d>250 \AA$ is a factor of five greater than the emission from the thinnest $50 \AA$ sample. This indicates that the majority of the emitted electrons originated in the bulk of the metal and not on the surface. The back $b$ coefficient initially decays exponentially, but then reaches a constant value of $\sim 0.2 \mathrm{pC} / \mu \mathrm{J}$ at $1000 \AA$. However, this charge was found to be present with no sample in place- - therefore it is background charge caused by scattered $217 \mathrm{~nm}$ light. 

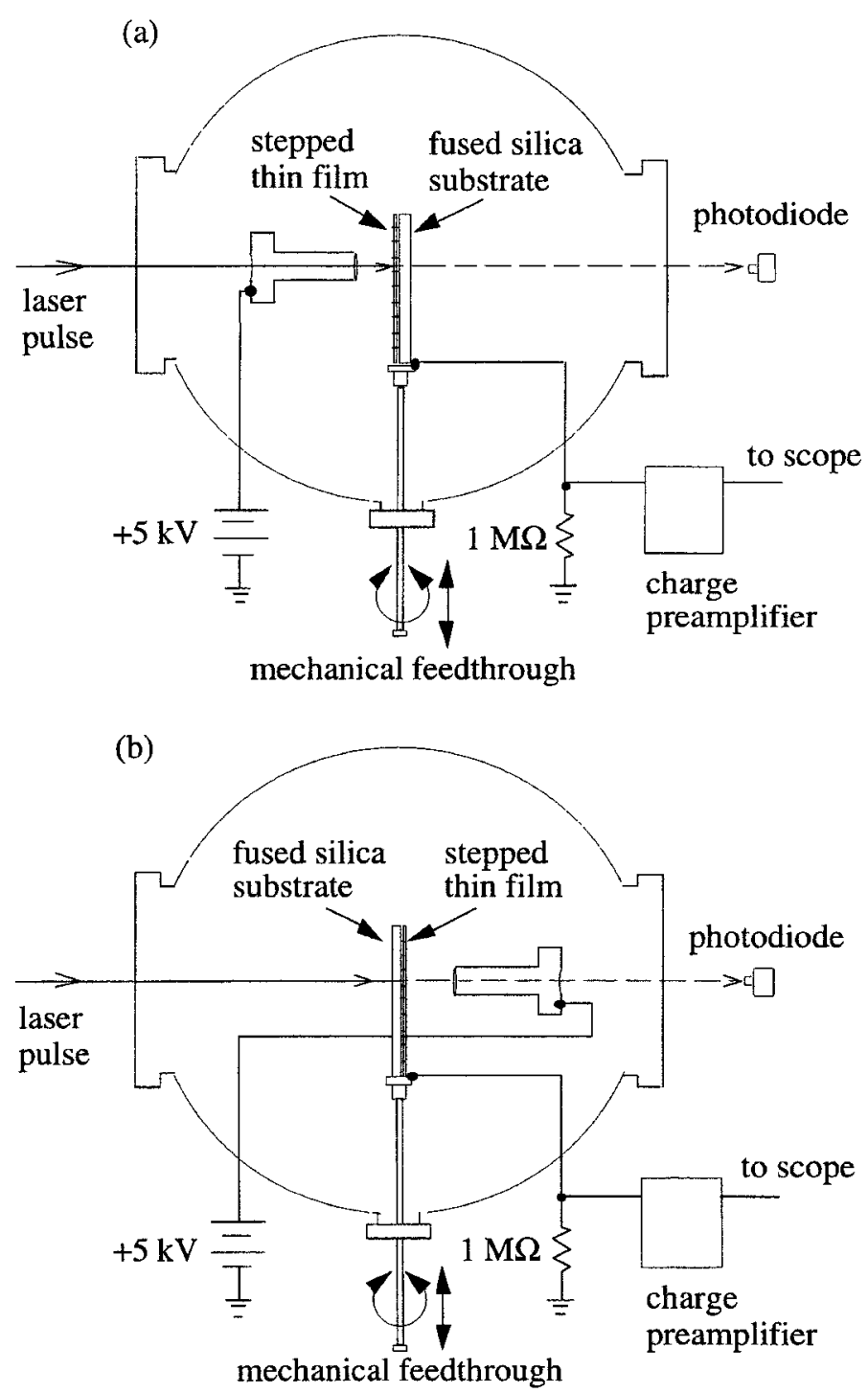

Figure 4: Setup for the multiphoton thin film illumination experiment: (a) front illumination configuration (b) back illumination configuration. 


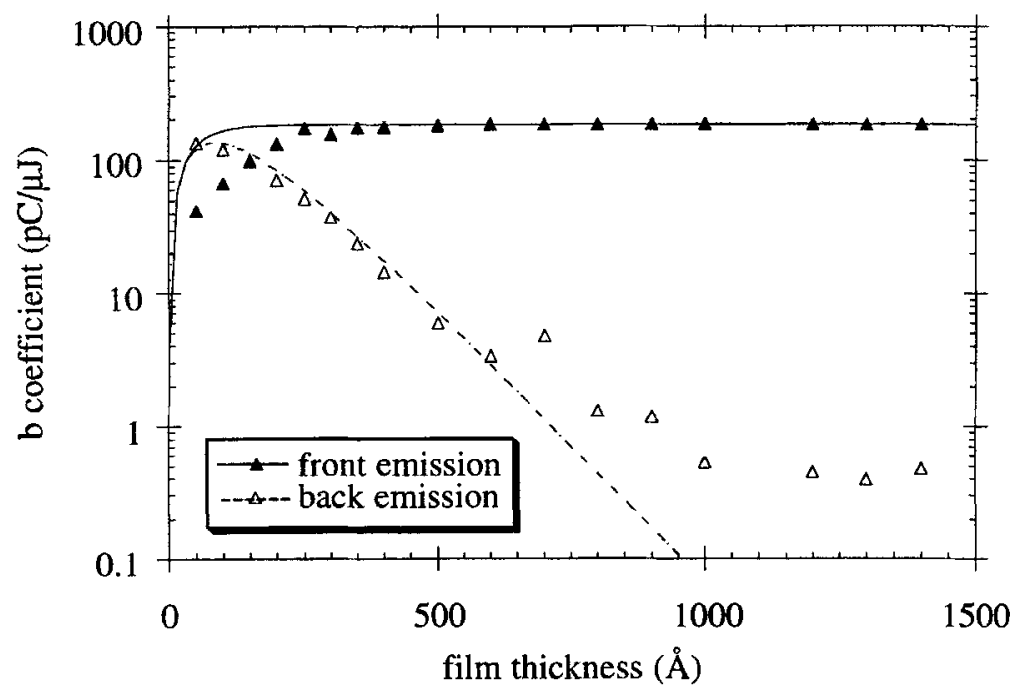

Figure 5: The measured electron yield ( $b$ coefficients) vs. copper film thickness for front and back illumination by $217 \mathrm{~nm}$ laser pulses, plotted with the theoretical curves. The maximum emission depth is $250 \AA$. 
An unexpected result shown in this plot is that for small thicknesses (50 and $100 \AA$ ) the back electron yield is greater than the front yield. However, this may be due to the presence of copper oxide or other contaminants on the metal surface. In the front illumination configuration the laser pulses are incident on the bare copper side; any surface contaminants may absorb some of the light and thereby reduce the energy available to the conduction electrons. In the back illumination configuration, on the other hand, the laser pulses are incident on the surface which has been deposited on the fused silica substrate. This surface is protected by the substrate and thus has no contaminants which may absorb the light. Therefore, for small thicknesses the back emission can be greater because the laser energy incident on the conduction electrons is higher.

\section{EMISSION PROMPTNESS}

The measurements of the maximum emission depth $d_{\max }$ can be used to obtain an upper limit on the time delay between an incident laser pulse and the resulting emitted electron bunch. Because the photoemitted electrons traveled ballistically to the metal surface, the maximum time delay will be the transit time for electrons from the maximum emission depth to reach the surface:

$$
\Delta t_{\max }=\frac{d_{\max }}{v_{e}}
$$

The minimum electron velocity $v_{e}$ for escape will be the Fermi velocity $v_{F}$ plus the velocity required to overcome the work function $v_{e \Phi}$ :

$$
v_{e}=v_{F}+v_{e \Phi}=\sqrt{\frac{2 \epsilon_{F}}{m_{e}}}+\sqrt{\frac{2 e \Phi}{m_{e}}}
$$

where $m_{e}$ is the electron mass. For copper $\epsilon_{F}=7.0 \mathrm{eV}$ and $e \Phi=4.6 \mathrm{eV}$, thus the minimum escape velocity is

$$
v_{e}=2.0 \times 10^{6} \mathrm{~m} / \mathrm{s}
$$

For the maximum escape depth $d_{\max }=250 \AA$ measured for the $217 \mathrm{~nm}$ emission, the delay time is

$$
\Delta t_{\max }=12 \mathrm{fs}
$$

This delay time is relatively small, and will not produce significant electron bunch broadening for incident laser pulses $>100 \mathrm{fs}$.

Another result from this work is that the electron yield from the back side of a thin copper film can be comparable to the yield from the front side of bulk copper. For example, the yield from the back emission of a $150 \AA$ film is only 
a factor of two less than the front bulk yield (Fig. 5). Thus a back illuminated photocathode could be used in DC guns coupled to a high frequency RF linac where laser beam access from the front side may be difficult or impossible. Such a photocathode would allow for synchronization to RF cycles or other fast events down the beam line (such as a beat-wave accelerator) which is not possible with conventional thermionic cathodes.

\section{References}

[1] S.M. Sze, J. L. Moll, and T. Sugano. Solid-State Electronics, volume 7, pages 509-23. Permagon Press, 1964.

[2] J. J. Quinn and R. A. Ferrell. Electron self-energy approach to correlation in a degenerate electron gas. Phys. Rev., 112(3):812-27, Nov. 1958.

[3] C.R. Cromwell, W.G. Spitzer, L.E. Howarth, and E.E. LaBate. Attenuation length measurements of hot electrons in metal films. Phys. Rev., 127(6):2006-15, Sep 1962.

[4] R.C. Weast, ed. Handbook of Chemistry and Physics. CRC Press, Boca Raton, Florida, 1984. 